\title{
Synthesis of 4-(1,3,5-Thiadiazinan-2-ylidene)-2-(3,4- dihydro-2H-1,3,5-thiadiazin-6-yl)pent-2-enedinitriles ${ }^{\dagger}$
}

\author{
Victor V. Dotsenko ${ }^{1,2,3, *}$, Elena A. Chigorina ${ }^{4}$ and Sergey G. Krivokolysko ${ }^{2,5}$ \\ 1 Department of Chemistry and High Technologies, Kuban State University, 149 Stavropolskaya str, \\ Krasnodar 350040, Russia \\ 2 ChemEx Lab, Vladimir Dal' Lugansk National University, 20A/7 Molodezhny, Lugansk 91034, Russia; \\ ksg-group-lugansk@mail.ru \\ 3 Department of Chemistry, North Caucasus Federal University, 1a Pushkin St., 355009 Stavropol, Russia \\ 4 The Federal State Unitary Enterprise "Institute of Chemical Reagents and High Purity Chemical \\ Substances" of National Research Centre "Kurchatov Institute", 3 Bogorodsky Val, Moscow 107076, Russia; \\ echigorina@mail.ru \\ * Correspondence: victor_dotsenko@bigmir.net \\ † Presented at the 22nd International Electronic Conference on Synthetic Organic Chemistry, 15 \\ November-15 December 2018; Available Online: https://sciforum.net/conference/ecsoc-22
}

Published: 14 November 2018

\begin{abstract}
The reaction of cyanothioacetamide with diethyl ethoxymethylenemalonate in the presence of triethylamine in hot $\mathrm{EtOH}$ proceeds non-selectively and leads to the formation of a mixture of triethylammonium 1,5-diamino-2,4-dicyano-5-thioxopenta-1,3-diene-1-thiolate (minor) and triethylammonium 3-cyano-5-ethoxycarbonyl-6-oxo-1H-pyridine-2-thiolate (major). Upon treatment with primary amines and $37 \%$ aqueous $\mathrm{HCHO}$ in boiling aqueous ethanol, the reaction product affords only 4-(1,3,5-thiadiazinan-2-ylidene)-2-(3,4-dihydro-2H-1,3,5-thiadiazin6-yl)pent-2-enedinitrile derivatives, instead of the expected pyrido[2,1-b][1,3,5]thiadiazines. Triethylammonium 3-cyano-5-ethoxycarbonyl-6-oxo-1H-pyridine-2-thiolate does not react under these conditions. The structure of the resulted products was confirmed by means of NMR, IR spectroscopy, and LCMS. The mechanism of the formation of the products is discussed.
\end{abstract}

Keywords: cyanothioacetamide; diethyl ethoxymethylenemalonate; aminomethylation; Mannich reaction; 1,3,5-thiadiazines

\section{Graphical Abstract}

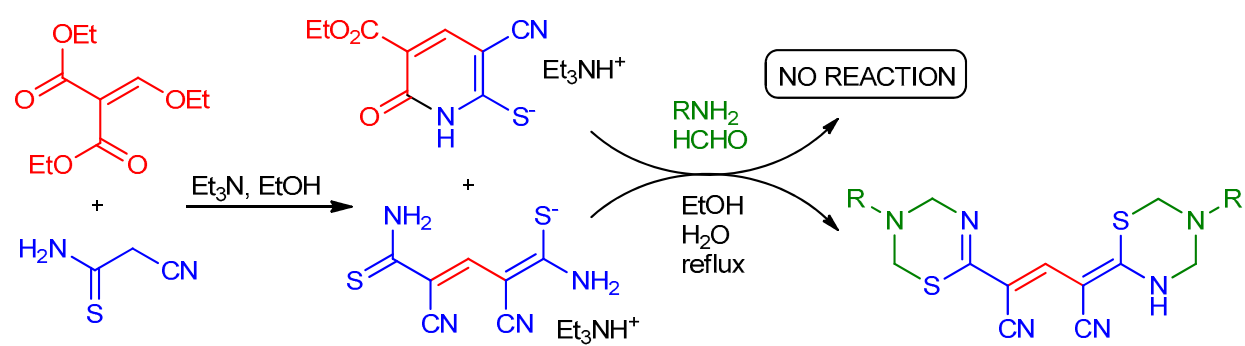

The Mannich reaction is an effective tool to build $\mathrm{C}-\mathrm{C}-\mathrm{N}$ and $\mathrm{X}-\mathrm{C}-\mathrm{N}$ bonds (where $\mathrm{X}=\mathrm{N}, \mathrm{O}, \mathrm{S}$, $\mathrm{Se}, \mathrm{P}$, etc.), and therefore it is often used in the synthesis of a wide variety of heterocyclic compounds (for the reviews on the various modifications of the Mannich reaction, see [1-9]. 1,3,5-Thiadiazines belong to an important class of heterocyclic compounds. The synthesis of 1,3,5-thiadiazines is successfully realized on the basis of Mannich-type aminomethylation [10-13]. 1,3,5-Thiadiazines are also of interest due a wide range of biological activity and practically important properties [12,14,15]; 
among the most important representatives of this class, the fungicide Dazomet and the highly effective insecticide Buprofezin, are worth mentioning (Figure 1).<smiles>CN1CSC(=S)N(C)C1</smiles>

Dazomet<smiles>CC(C)N1CN(c2ccccc2)CS/C1=N\C(C)(C)C</smiles>

Buprofezin

Figure 1. Bioactive 1,3,5-thiadiazines.

Our interest in the synthesis of condensed 1,3,5-thiadiazines through the aminomethylation of cyclic thioamides [12,16-18] prompted us to study the behavior of new cyclic cyanothioacetamide derivatives in the reaction with primary amines and formaldehyde. Triethylammonium 3-cyano-5-ethoxycarbonyl-6-oxo-1H-pyridine-2-thiolate $\mathbf{1}$ was chosen as a starting compound. The synthesis of $\mathrm{N}$-methylmorpholinium analogue $\mathbf{2}$ in moderate yield (52\%) was described in Yakunin, Dyachenko, and Litvinov [19] (Scheme 1).<smiles>CCOC=C(C(=O)OCC)C(=O)OCC</smiles>

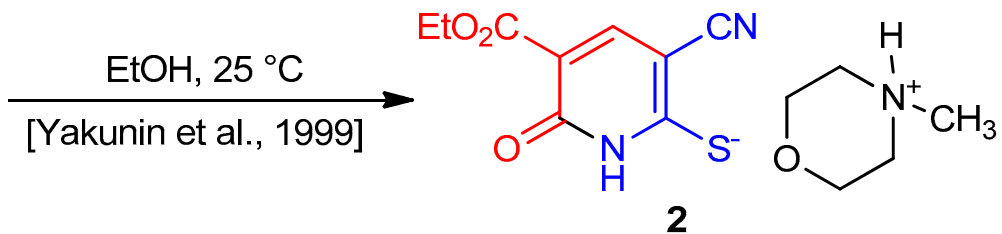

Scheme 1. Synthesis of thiolate 2 .

Due to the presence of two nucleophilic centers (endocyclic nitrogen atom and sulfur atom), thiolate $\mathbf{1}$ seemed to be a promising substrate for aminomethylation leading to pyrido[2,1-b][1,3,5]thiadiazines with potential biological activity $[12,16]$. In the aminomethylation reactions, the nature of the trialkylammonium cation, as a rule, does not affect significantly the yields and the direction of a process. Therefore, in order to increase the yield of the starting thiolate, we decided to replace $\mathrm{N}$-methylmorpholine with a more basic [20] triethylamine.

To prepare thiolate 1, cyanothioacetamide was reacted with ethoxymethylene malonate in the presence of a 1.5-fold excess of $\mathrm{Et}_{3} \mathrm{~N}$ while slowly heating the reaction mixture to boiling point. The reaction product was put into reaction with primary amines with an excess of $37 \%$ without further purification. The aminomethylation products were precipitated from a boiling solution during the reaction. To our surprise, the IR spectra of the supposed pyrido[2,1-b][1,3,5] thiadiazines 3 revealed the absence of stretching vibration bands of the ester $\mathrm{C}=\mathrm{O}$ group, while the bands corresponding to the stretching vibrations of the conjugated cyano group $\left(\sim 2215-2220 \mathrm{~cm}^{-1}\right)$ and $\mathrm{N}-\mathrm{H}$ bonds $(v \sim$ 3350-3400 $\mathrm{cm}^{-1}$ ) were observed. In the ${ }^{1} \mathrm{H}$ NMR spectra of the synthesized compounds, there are a broadened $\mathrm{NH}$ proton singlet $(\delta 11.86-12.06 \mathrm{ppm})$, two sets of signals from primary amines, and four non-equivalent signals of the $\mathrm{X}-\mathrm{CH}_{2}-\mathrm{N}$ protons in the expected region ( $\delta 4.5 \ldots 6.0 \mathrm{ppm}$ ), as well as a narrow singlet at $\delta 7.63-7.85 \mathrm{ppm}$ (Figure 2). Moreover, the characteristic signals of the ethoxycarbonyl group were absent in the NMR spectrum. Analysis of spectral data, the results of elemental analysis, as well as HPLC-MS data allowed us to conclude that the products actually have a structure of 4-(1,3,5-thiadiazin-2-ylidene)-2-(3,4-dihydro-2H-1,3,5-thiadiazin-6-yl)pent-2-endinitriles 4 (Scheme 2). A detailed analysis of the spectral data of the starting thiolate 1 revealed that, unlike the 
thiolate 2 obtained by a known method [19], the sample we synthesized is actually a mixture of triethylammonium 1,5-diamino-2,4-dicyano-5-thioxopenta-1,3-diene-1-thiolate 5 (minor product) and triethylammonium 3-cyano-5-ethoxycarbonyl-6-oxo-1H-pyridine-2-thiolate 1 (main product) (Scheme 2).

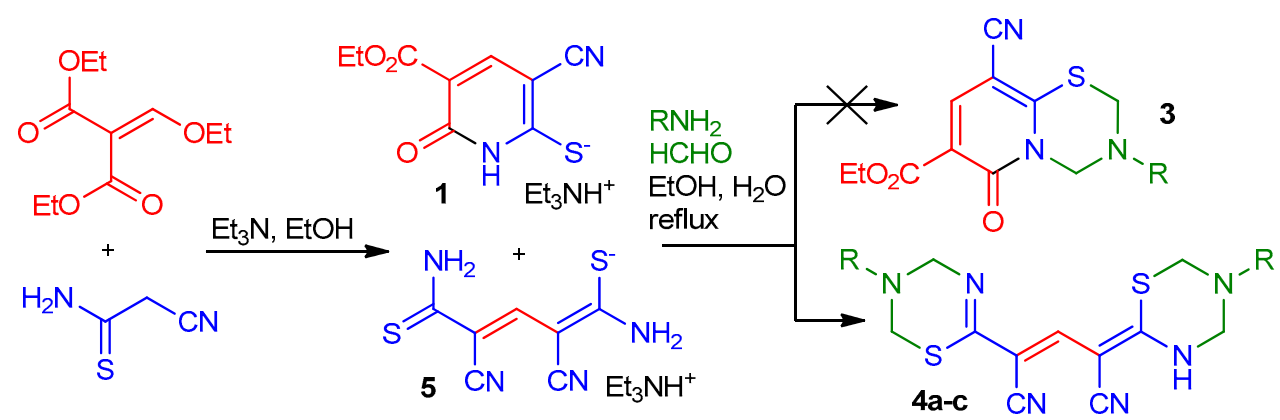

4 a: $\mathrm{R}=\mathrm{CH}_{2} \mathrm{Ph} ; \mathbf{b}: \mathrm{R}=4-\mathrm{EtOC}_{6} \mathrm{H}_{4} ; \mathrm{c}: \mathrm{R}=4-\mathrm{EtC}_{6} \mathrm{H}_{4}$.

Scheme 2. Synthesis of compounds $4 a-c$.

These results can be explained by the mechanism presented in Scheme 3. First, an $\mathrm{S}_{\mathrm{N}} \mathrm{Vin}$ vinyl nucleophilic substitution reaction takes place [21-23] leading to the formation of an intermediate diene 6 . The formation of such diene species in the reactions of vinyl ethers with active methylene compounds is well documented [24-28]. Next, presumably, the activated double bond of intermediate 6 was attacked by cyanothioacetamide anion, followed by decomposition of the Michael adduct 7, which is accompanied by elimination of malonic ester and formation of pentadiene thiolate 5 . The formation of such structures was previously observed in the reaction of cyanoacetanilides with ethoxymethylenemalonate [28]. At the same time, an alternative and preferential pathway for the transformation of intermediate 6 is intramolecular 6-exo-trig-cyclization with the formation of pyridine-2-thiolate 1.

Further investigation of the reaction of cyanothioacetamide with ethoxymethylene malonate in the presence of triethylamine and N-methylmorpholine allowed us to conclude that: (1) in the case of N-methylmorpholine under the conditions described earlier [19]; pentadiethiolates 5 were not isolated; (2) replacement of $\mathrm{N}$-methylmorpholine to triethylamine favors the formation of compound $\mathbf{5}$ under short-term heating of a mixture; (3) the content of product $\mathbf{5}$ in the mixture does not exceed $10-15 \mathrm{~mol} \%$ (according to ${ }^{1} \mathrm{H}$ NMR); (4) analytically pure triethylammonium 3-cyano-5-ethoxycarbonyl-6-oxo-1H-pyridine-2-thiolate $\mathbf{1}$ can be prepared by reaction of cyanothioacetamide and ethoxymethylene malonate in acetonic solution at the room temperature; (5) a longer heating neither favors the formation of compound 5, nor leads to an increase in the yields of thiolates $\mathbf{1}$ or $\mathbf{2}$, but leads to a noticeable resinification of the reaction mixture, possibly due to further intramolecular cyclizations of compounds 5 or 7 , or self-condensation of cyanothioacetamide, which occurs under basic conditions $[29,30]$. 

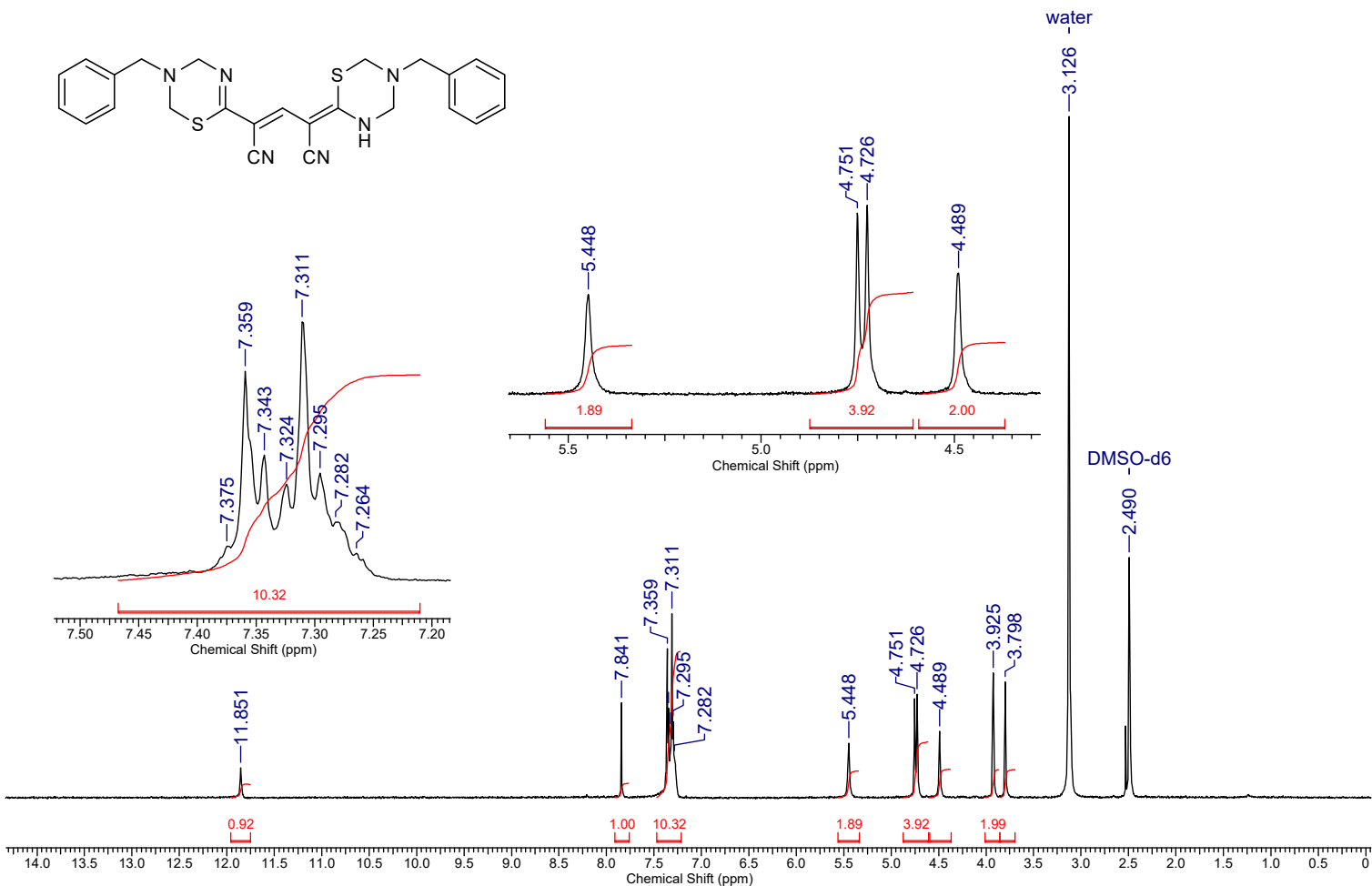

Figure 2. ${ }^{1} \mathrm{H}$ NMR spectrum (400 MHz, DMSO-d6) of 4-(5-benzyl-1,3,5-thiadiazin-2-ylidene)-2(3-benzyl-3,4-dihydro-2H-1, 3,5-thiadiazin-6-yl)pent-2-endinitrile 4a.

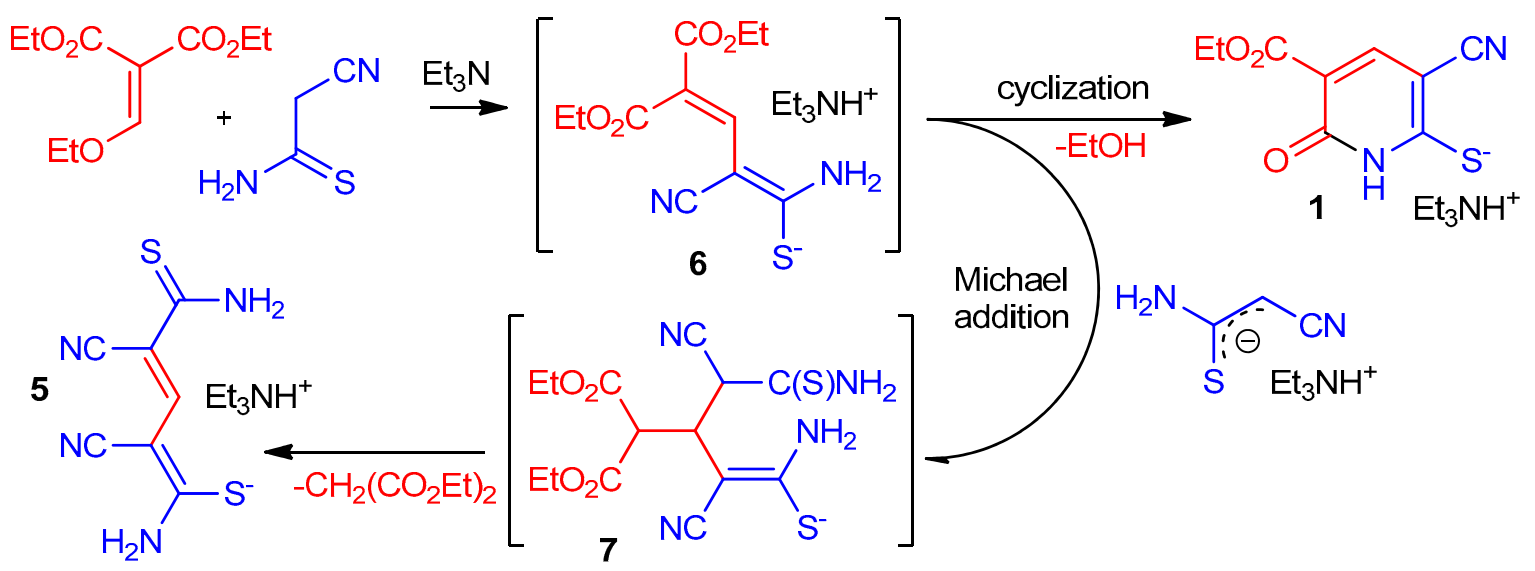

Scheme 3. The possible mechanism of the formation of compounds $\mathbf{1}$ and $\mathbf{5}$

It should also be noted that, contrary to expectations, neither pure thiolate $\mathbf{1}$ nor thiolate $\mathbf{2}$ give any Mannich-type products under the conditions stated; a noticeable resinification of reaction mass was observed during the synthesis. The TLC and NMR data of a gummy residue after removal of the solvent showed the presence of starting thiolate, along with a complex mixture of the reaction products between amines, $\mathrm{HCHO}$, and solvent. In our opinion, this can be explained by the reduced $\mathrm{N}$-/S-nucleophilicity of the substrate due to the presence of two strong electron-withdrawing groups $(\mathrm{CN}, \mathrm{COOEt})$ in the pyridine ring.

\section{Experiment}

IR spectra were recorded on an IKS-29 spectrometer in KBr pellets. ${ }^{1} \mathrm{H}$ NMR spectra were recorded on a Bruker DPX-400 spectrometer (400 MHz) in DMSO-d6 using TMS as an internal standard. HPLC-MS analysis was performed on a Shimadzu LC-10AD LC with a Shimadzu SP D-10A UV-Vis (254 nm) detector and Sedex 75 ELSD, combined with a PE SCIEX API 150EX mass spectrometer and atmospheric pressure electrospray ionization. Selected experimental procedures 
will now be described. Cyanothioacetamide was synthesized by passing hydrogen sulfide through a solution of malononitrile in EtOH according to a known procedure [31]. Ethoxymethylenemalonic ester is a commercially available reagent (Acros).

4-(5-Benzyl-1,3,5-thiadiazin-2-ylidene)-2-(3-benzyl-3,4-dihydro-2H-1,3,5-thiadiazin-6-yl)pent-2-endinitrile $4 \mathbf{a}$.

A mixture of thiolates 1 and 5 (1.0 g) (prepared by reaction of cyanothioacetamide with ethoxymethylene malonate in the presence of $\mathrm{Et}_{3} \mathrm{~N}$ in boiling $\left.\mathrm{EtOH}\right)$, was dissolved in hot $80 \% \mathrm{EtOH}$ $(10 \mathrm{~mL})$. The resulting solution was filtered through a paper filter, then the filtrate was added to a solution of benzylamine $(3 \mathrm{mmol})$ and an excess of formalin $(5 \mathrm{~mL})$ in $5 \mathrm{~mL}$ of EtOH. The mixture was boiled with stirring for 1-2 min, during which time a crystalline precipitate formed. The product was filtered off and washed with EtOH to afford 1,3,5-thiadiazine 4a in analytically pure form.<smiles>N#CC(/C=C(\C#N)C1=NCN(Cc2ccccc2)CS1)=C1/NCN(Cc2ccccc2)CS1</smiles>

Yellow cryastalline solid, yield was $0.18 \mathrm{~g}$. IR (nujol), v, $\mathrm{cm}^{-1}: 3360(\mathrm{~N}-\mathrm{H}) ; 2217(\mathrm{CN}) . \mathrm{NMR}^{1} \mathrm{H}$ (400 MHz, DMSO-d $d_{6}$ ), $\delta$, ppm: 3.80 (br s, $2 \mathrm{H}, \mathrm{NC}_{2} \mathrm{Ph}$ ); 3.93 (br s, $2 \mathrm{H}, \mathrm{NC}_{2} \mathrm{Ph}$ ); 4.49 (br s, $2 \mathrm{H}$, $\mathrm{NC}_{2} \mathrm{NH}$ ); 4.73 (br s, $2 \mathrm{H}, \mathrm{NC} \underline{\mathrm{H}}_{2} \mathrm{~S}$ ); 4.75 (br s, $2 \mathrm{H}, \underline{\mathrm{NC}}_{2} \mathrm{~S}$ ); 5.45 (br s, $2 \mathrm{H}, \mathrm{NC}_{2} \underline{\mathrm{H}}_{2} \mathrm{~N}$ ); 7.26-7.38 (m, 10 $\mathrm{H}, 2 \mathrm{Ph}$ ); 7.84 (s, $1 \mathrm{H},-\mathrm{CH}=) ; 11.85$ (br s, $1 \mathrm{H}, \mathrm{NH}$ ). LC-MS (ESI), m/z: $947.0[2 \mathrm{M}+\mathrm{H}]^{+}, 826.5$ $\left[2 \mathrm{M}+\mathrm{H}-\mathrm{PhCH}_{2} \mathrm{~N}=\mathrm{CH}_{2}\right]^{+}, 473.5[\mathrm{M}+\mathrm{H}]^{+}, 354.5\left[\mathrm{M}-\mathrm{PhCH}_{2} \mathrm{~N}=\mathrm{CH}_{2}+\mathrm{H}\right]^{+}, 235.1\left[\mathrm{M}-2 \mathrm{PhCH}_{2} \mathrm{~N}=\mathrm{CH}_{2}+\mathrm{H}\right]^{+}$. Found, \%: C 63.64; $\mathrm{H}$ 5.22; N 17.72. $\mathrm{C}_{25} \mathrm{H}_{24} \mathrm{~N}_{6} \mathrm{~S}_{2}(\mathrm{M}=472.63)$. Calculated, \%: C 63.53; H 5.12; $\mathrm{N} 17.78$.

\section{References}

1. Akhmetova, V.R.; Nadyrgulova, G.R.; Niatshina, Z.T.; Dzhemilev, U.M. Cyclothiomethylation of primary amines with formaldehyde and hydrogen sulfide to nitrogen-and sulfur-containing heterocycles. Chem. Heterocyclic Compd. 2009, 45, 1155-1176.

2. Akhmetova, V.R.; Rakhimova, E.B. One-pot cyclothiomethylation of amines as efficient method for the synthesis of saturated five-, six-, seven-, and eight-membered S,N-Heterocycles. Russ. J. Organic Chem. 2014, 50, 1711-1731.

3. Arend, M.; Westermann, B.; Risch, N. Modern variants of the Mannich reaction. Angeww. Chem. Int. Ed. 1998, 37, 1044-1070.

4. Bur, S.K.; Martin, S.F. Vinylogous Mannich reactions: Selectivity and synthetic utility. Tetrahedron 2001, 57, 3221-3242.

5. Keglevich, G.; Bálint, E. The Kabachnik-Fields reaction: Mechanism and synthetic use. Molecules 2012, 17, $12821-12835$.

6. Roselló, M.S.; del Pozo, C.; Fustero, S. A Decade of Advance in the Asymmetric Vinylogous Mannich Reaction. Synthesis 2016, 48, 2553-2571.

7. Subramaniapillai, S.G. Mannich reaction: A versatile and convenient approach to bioactive skeletons. J. Chem. Sci. 2013, 125, 467-482.

8. Tramontini, M. Advances in the chemistry of Mannich Bases. Synthesis 1973, 12, 703-775.

9. Tramontini, M.; Angiolini, L. Further advances in the chemistry of Mannich bases. Tetrahedron 1990, 46, 1791-1837.

10. Akhmetova, V.R.; Rakhimova, E.B.; Galimzyanova, N.F.; Ibragimov, A.G. Synthesis and biological activity of five-, six-and seven-membered S, N-containing saturated heterocycles, Chap 5. Bioactive heterocycles: Synthesis and biological evaluation. Nova Sci. Pub. Incl. Hauppauge 2012, 5, 98-115.

11. Bermello, J.C.; Piñeiro, R.P.; Fidalgo, L.M.; Cabrera, H.R.; Navarro, M.S. Thiadiazine derivatives as antiprotozoal new drugs. Open Med. Chem. J. 2011, 5, 51.

12. Dotsenko, V.V.; Frolov, K.A.; Krivokolysko, S.G. Synthesis of partially hydrogenated 1,3,5-thiadiazines by Mannich reaction. Chem. Heterocyclic Compd. 2015, 51, 109-127.

13. Rodríguez, H.; Suárez, M.; Albericio, F. Thiadiazines, N, N-heterocycles of biological relevance. Molecules 2012, 17, 7612-7628. 
14. Kanno, H. An approach to a novel insect growth regulator buprofezin (Applaud). Pure Appl. Chem. 1987, $59,1027-1032$.

15. De Cock, A.; Degheele, D. Buprofezin: A novel chitin synthesis inhibitor affecting specifically planthoppers, whiteflies and scale insects. In Insecticides with Novel Mode of Action: Mechanism and Application; Ishaaya, I., Degheele, D., Eds.; Springer: New York, NY, USA, 1998; pp. 74-91.

16. Bibik, E.Y.; Yaroshevskaya, O.G.; Devdera, A.V.; Demenko, A.V.; Zakharov, V.V.; Frolov, K.A.; Dotsenko, V.V.; Krivokolysko, S.G. Search for Anti-Inflammatory Agents in the Tetrahydropyrido[2,1-b][1,3,5]Thiadiazine Series. Pharm. Chem. J. 2017, 51, 648-651.

17. Chigorina, E.A.; Frolov, K.A.; Dotsenko, V.V.; Goloveshkin, A.S.; Bushmarinov, I.S.; Krivokolysko, S.G. Synthesis and structure of new 3,7-diazabicyclo[3.3.1]nonane derivatives. Russ. Chem. Bull. 2016, 65, 2260-2269.

18. Dotsenko, V.V.; Frolov, K.A., Krivokolysko, S.G.; Chigorina, E.A.; Pekhtereva, T.M.; Suykov, S.Y.; Papayanina, E.S.; Dmitrienko, A.O.; Bushmarinov, I.S. Aminomethylation of morpholinium and N-methylmorpholinium 3,5-dicyano-4,4-dimethyl-6-oxo-1,4,5,6-tetrahydropyridine-2-thiolates. Chem. Heterocyclic Compd. 2016, 52, 116-127.

19. Yakunin, Y.Y.; Dyachenko, V.D.; Litvinov, V.P. Synthesis of 3-cyano-5-ethoxycarbonyl-6-hydroxypyridine-2-thiol derivatives. Russ. Chem. Bull. 1999, 48, 195-196.

20. Rayer, A.V.; Sumon, K.Z.; Jaffari, L.; Henni, A. Dissociation Constants (pKa) of Tertiary and Cyclic Amines: Structural and Temperature Dependences. J. Chem. Eng. Data 2014, 59, 3805-3813.

21. Dyachenko, V.D.; Tkachev, R.P. Functionally-substituted alkoxyethylenes in reactions with nucleophiles. Part I. Synthesis of six-membered heterocycles. Russ. J. Organic Chem. 2003, 39, 757-793.

22. Dyachenko, V.D.; Tkachev, R.P. Functionally-substituted alkoxyethylenes in reactions with nucleophiles: Part 2. Synthesis of noncyclic structures, benzene derivatives, 5-, 7-membered, and macroheterocycles. Russ. J. Organic Chem. 2006, 42, 149-171.

23. Kudyakova, Y.S.; Bazhin, D.N.; Goryaeva, M.V.; Burgart, Y.V.; Saloutin, V.I. The use of 2-(1-alkoxyalkylidene)-1, 3-dicarbonyl compounds in organic synthesis. Russ. Chem. Rev. 2014, 83, 120.

24. Dyachenko, V.D.; Tkachev, R.P. 3-Amino-3-thioxopropanamide in the synthesis of functionally substituted nicotinamides. Russ. J. Organic Chem. 2003, 39, 1174-1179.

25. Dyachenko, V.D.; Tkachev, R.P.; Chernega, A.N. Heterocyclization of 1, 3-butadienethiolates. Chem. Heterocyclic Compd. 2005, 41, 503-510.

26. Schmidt, H.W.; Junek, H. Zur Synthese von Alkoxymethylenmalonitrilen, Tetracyanpropeniden und hochsubstituierten $\alpha$-Aminopyridinen. Monatshefte für Chemie 1977, 108, 895-900.

27. Tkachev, R.P.; Bityukova, O.S.; Dyachenko, V.D.; Tkacheva, V.P.; Dyachenko, A.D. Competing processes in the condensation of diethyl ethoxymethylidenemalonate and malononitrile derivatives with $\mathrm{CH}$ acids containing a thiocarbamoyl group. Russ. J. Gen. Chem. 2007, 77, 116-123.

28. Tkachova, V.P.; Gorobets, N.Y.; Tkachov, R.P.; Dyachenko, O.D.; Rusanov, E.B.; Dyachenko, V.D. Reactions of diethyl 2-(ethoxymethylene)malonate with 2-cyanoacetanilides: Unexpected transfer of the ethoxymethylene moiety. ARKIVOC 2010, 11, 254-264.

29. Fahmy, S.M.; Mohareb, R.M. Cyanothioacetamide in heterocyclic synthesis: A novel synthesis of 2-pyridothione derivatives. Tetrahedron 1986, 42, 687-690.

30. Mohareb, R.M.; Fahmy, S.M. Cyanothioacetamide in heterocyclic synthesis: A new approach for the synthesis of 2-pyridothione and 2-pyridazinothione derivatives. Zeitschrift für Naturforschung B 1986, 41, 105-109.

31. Brunskill, J.S.; De, A.; Ewing, D.F. Dimerisation of 3-aryl-2-cyanothioacrylamides. A [2s+4s]cycloaddition to give substituted 3,4-dihydro-2H-thiopyrans. J. Chem. Soc. Perkin Trans. 1978, 1, 629-633.

(C) 2019 by the authors. Licensee MDPI, Basel, Switzerland. This article is an open access article distributed under the terms and conditions of the Creative Commons Attribution (CC BY) license (http://creativecommons.org/licenses/by/4.0/). 